\title{
Balanced Avoidance Games on Random Graphs
}

\author{
Martin Marciniszyn, Dieter Mitsche and Miloš Stojaković ${ }^{\dagger}$
}

Institute of Theoretical Computer Science, ETH Zurich, CH-8092 Zurich, Switzerland

We introduce and study balanced online graph avoidance games on the random graph process. The game is played by a player we call Painter. Edges of the complete graph with $n$ vertices are revealed two at a time in a random order. In each move, Painter immediately and irrevocably decides on a balanced coloring of the new edge pair: either the first edge is colored red and the second one blue or vice versa. His goal is to avoid a monochromatic copy of a given fixed graph $H$ in both colors for as long as possible. The game ends as soon as the first monochromatic copy of $H$ has appeared.

We show that the duration of the game is determined by a threshold function $m_{H}=m_{H}(n)$. More precisely, Painter will asymptotically almost surely (a.a.s.) lose the game after $m=\omega\left(m_{H}\right)$ edge pairs in the process. On the other hand, there is an essentially optimal strategy, that is, if the game lasts for $m=o\left(m_{H}\right)$ moves, then Painter will a.a.s. successfully avoid monochromatic copies of $H$ using this strategy. Our attempt is to determine the threshold function for certain graph-theoretic structures, e.g., cycles.

\section{Introduction}

The game we investigate is played by a single player called Painter. He maintains a balanced 2-coloring of the edges in the random graph process. The game begins on an empty graph with $n$ vertices. The edges are revealed in the order chosen uniformly at random among all $\left(\begin{array}{l}n \\ 2\end{array}\right)$ ! permutations. In each step, the next two edges are presented to Painter, who has to assign a balanced 2-coloring to them immediately. He may either color the first edge red and the second one blue or vice versa. Since Painter has no knowledge about the future edges, we call this a balanced online coloring. His goal is to avoid a monochromatic copy of some fixed graph $H$ in both colors for as long as possible. We call this the balanced online $H$-avoidance game.

Let $e_{1}, e_{2}, \ldots, e_{M}, M=\left(\begin{array}{c}n \\ 2\end{array}\right)$, be the labeled edges of the complete graph $K_{n}$, and let $\pi:[M] \rightarrow[M]$ be a permutation of the set $[M]$ chosen uniformly at random. By $\left(G_{i}\right)_{i=1}^{M}$ we denote the sequence of graphs on $n$ vertices with the edge-set $E\left(G_{i}\right)=\left\{e_{\pi(1)}, e_{\pi(2)}, \ldots, e_{\pi(i)}\right\}$. In the $m$-th move of the game, Painter is presented with the edges $e_{\pi(2 m-1)}$ and $e_{\pi(2 m)}$ and has to color one of them red and the other one blue. Therefore, after playing the first $m$ moves, Painter has created a balanced 2-coloring of the graph $G_{2 m}$. The duration of the game is the index of the move, in which the first monochromatic copy of $H$ is created, in either red or blue.

\footnotetext{
†Supported by the joint Berlin-Zurich graduate program "Combinatorics, Geometry, and Computation", financed by the German Science Foundation (DFG) and ETH Zurich.
}

1365-8050 (c) 2005 Discrete Mathematics and Theoretical Computer Science (DMTCS), Nancy, France 
Since the game is played on the random graph process, we can reason about the probability of the event $\mathcal{P}(n, m)=$ "Painter loses in the first $m$ moves", assuming $n$ and $m$ are fixed. We say that this event occurs asymptotically almost surely (a.a.s.) if $\lim _{n \rightarrow \infty} \operatorname{Pr}[\mathcal{P}(n, m)]=1$. In the present paper, we attempt to determine a threshold function $m_{H}=m_{H}(n)$ for a fixed graph $H$, such that $\mathcal{P}(n, m)$ occurs a.a.s. if $m=\omega\left(m_{H}\right)$, but for $m=o\left(m_{H}\right)$, we shall provide a winning strategy for Painter, i.e.,

$$
\lim _{n \rightarrow \infty} \operatorname{Pr}[\mathcal{P}(n, m)]=\left\{\begin{array}{ll}
1 & \text { if } m=\omega\left(m_{H}\right) \\
0 & \text { if } m=o\left(m_{H}\right)
\end{array} .\right.
$$

We are interested in determining this threshold for a number of graph-theoretic structures, like cycles, stars, and paths.

Motivation and known results. In [4], Friedgut et al. introduce the concept of an online game played on the random graph process. In this game the player is 2-coloring edges, one at the time in an online fashion, not caring about the balancedness of the coloring. His goal is to avoid a monochromatic copy of a fixed graph $H$ for as long as possible. In [4], the question of how long the player can play the triangle game, $H=K_{3}$, is resolved.

Extending this result, Marciniszyn, Spöhel and Steger [5] analyze the game of avoiding monochromatic cliques $H=K_{\ell}$ of any fixed size $\ell$, and they exhibit a threshold for the number of moves at which the player loses a.a.s. It turns out that the near-optimal strategy is to play greedily-using the first color whenever possible, and the second one only to prevent losing immediately. The colorings obtained by following this strategy are typically unbalanced. A natural question arising is: if the player is forced to keep his coloring balanced, how long can he survive without losing? We try to give an answer to this question by looking at an analogous game, in which the coloring of the graph is balanced. As it turns out, several thresholds we obtain in the balanced game are not the same as for the unbalanced game, showing that the balancedness condition makes a substantial difference. For instance, applying a general criterion from [5] to cycles $C_{\ell}$ of length $\ell$ yields the threshold $m_{H}=n^{(\ell+1) / \ell}$ in the unbalanced case, whereas we can derive the threshold $m_{H}=n^{2 \ell /(2 \ell-1)}$ from our results for balanced online colorings (see Corollary 3). Hence, the balanced online cycle avoidance game will end substantially earlier than the unbalanced game.

Another motivation comes from Beck's Chooser-Picker games on graphs [2,3]. The game is played by two players, Chooser and Picker, on the edges of the complete graph $K_{n}$. In each move, Picker picks two uncolored edges, and then Chooser chooses which of them to color red, and which blue. That way, a balanced coloring of the subset of edges of $K_{n}$ is maintained. In the "misère" version of the game, Chooser wins if at the end of the game (when all edges are colored) there is no red copy of a fixed graph $H$. Otherwise, Picker wins. If Picker plays randomly, this game is quite similar to the balanced avoidance games that we introduce in the present paper. The only difference is that in our game we investigate a Ramsey-type [6] property, where $H$ has to be avoided in both colors.

Generally speaking, studying games on graphs and random graphs in parallel often uncovers surprising connections between thresholds for winning a game on one side, and thresholds for certain properties of random graphs on the other-see [1] and [8], where this phenomenon is pointed out for Maker-Breaker games on graphs. We also encounter some relations of this kind while dealing with balanced avoidance games. 


\section{Results}

\subsection{A criterion}

We present a general criterion that gives upper and lower bounds for the threshold $m_{H}$, provided that the graph $H$ satisfies certain conditions. In some cases the upper and lower bounds match and this criterion gives the exact value for $m_{H}$, like for the game of avoiding a monochromatic cycle of an arbitrary fixed length.

First, we give a general lower bound for the balanced game of avoiding a graph having certain properties. By $e_{H}$ and $v_{H}$ we denote the number of edges and vertices of $H$, respectively. A graph $H$ is called balanced if for all subgraphs $H^{\prime} \subseteq H, e_{H^{\prime}} / v_{H^{\prime}} \leq e_{H} / v_{H}$.

Theorem 1 Let $H$ be a graph with $v_{H} \geq 2$. If every subgraph of $H$ with $e_{H}-1$ edges is balanced, then the balanced online $H$-avoidance game a.a.s. lasts for $m(n)=o\left(n^{2-1 / \underline{m}(H)}\right)$ moves, where

$$
\underline{m}(H)=\max \left\{\frac{2 e_{F}-1}{2 v_{F}-2}: F \subseteq H \text { and } v_{F} \geq 2\right\} .
$$

The proof is performed by analyzing a greedy strategy. The number of copies of any subgraph $H^{-} \subseteq H$ with $e_{H^{-}}=e_{H}-1$ can be bounded from above with exponentially small error probability [9]. In general, there are two ways for Painter to lose the game in the $i$-th move - if he has to color two edges each of which completes a red (or blue respectively) copy of $H^{-}$to $H$, or if one of the edges closes both a red and blue copy of a $\mathrm{H}^{-}$. We estimate the number of both types of threats simultaneously for all possible graphs $H^{-}$in order to conclude that Painter will a.a.s. never encounter such a threat.

Next, we give an upper bound for $m_{H}$, which also requires certain properties of $H$.

Theorem 2 Let $H$ be a graph with $v_{H} \geq 3$ and $2 e_{H}>v_{H}$. If there exists a subgraph $H^{-} \subseteq H$ with $e_{H}-1$ edges such that for its 2-density we have

$$
\frac{2 e_{H}-1}{2 v_{H}-2} \geq m_{2}\left(H^{-}\right),
$$

then Painter will a.a.s. lose the balanced online $H$-avoidance game after $m(n)=\omega\left(n^{2-\left(2 v_{F}-2\right) /\left(2 e_{F}-1\right)}\right)$ moves, regardless of his strategy.

We prove this theorem by using the two round exposure technique - we divide the game into two rounds, $m=m_{1}+m_{2}$, where $m_{1}=C n^{2-\left(2 v_{H}-2\right) /\left(2 e_{H}-1\right)}$ for some large constant $C>0$. Then we apply a theorem of Rödl and Ruciński [7] to the coloring after the first round $G_{2 m_{1}}$ and obtain that for a constant fraction of all copies of $H^{-}$in $G_{2 m_{1}}$, these copies are in fact monochromatic and enforce a certain color on one vertex pair. Then, for the sake of a lower bound on the number of threats, it remains to prove that not too many of these copies share the same edge that makes them isomorphic to $H$. Since there are so many dangerous spots after the first round, Painter will create a monochromatic copy of $H$ in the second round a.a.s.

For some graphs the values for the upper and lower bound match and give rise to a threshold function. Combining Theorem 1 and Theorem 2, we obtain the threshold function

$$
m_{H}(n)=n^{2-\frac{2 v_{H}-2}{2 e_{H}-1}} .
$$

In particular, all requirements of both theorems hold for cycles $C_{\ell}$ of length $\ell$. 
Corollary 3 For any fixed integer $\ell \geq 3$, the duration of the balanced online $C_{\ell}$-avoidance game is determined by the threshold

$$
m_{C_{\ell}}(n)=n^{2-\frac{2 \ell-2}{2 \ell-1}}
$$

\subsection{Star game and path game}

The criterion from the last section does not give very good bounds on the threshold for avoiding trees in the balanced game. Here we give some better bounds for stars and paths.

Theorem 4 Let $\alpha>0$ be a constant, $k$ an integer, and $m=\Theta\left(n^{1-\alpha}\right)$.

(i) After $m^{\prime}=\omega(m)$ moves of the balanced game, Painter has created $\omega\left(m^{2 k-1} n^{-2 k+2}\right)$ monochromatic stars of size $k$ a.a.s.

(ii) For $m^{\prime \prime}=o(m)$, there is a strategy for Painter that enables him to create $o\left(m^{2 k-1} n^{-2 k+2}\right)$ monochromatic stars of size $k$ in the first $m^{\prime \prime}$ moves of the balanced game a.a.s.

The proof of $(i)$ is performed by analyzing the random graph created after $m^{\prime}$ moves. Namely, every $2 k+1$ star in this graph contains a monochromatic $k$ star, independent of Painter's strategy.

In the (ii) part, we exhibit a strategy and prove that it is optimal. If Painter is to color edges $v_{1} v_{2}$ and $v_{3} v_{4}$ in his $i$ th move, he spots the largest monochromatic star that is centered at one of vertices $v_{1}, \ldots, v_{4}$ at that moment. Note that there may be more than one star with that property in which case one of them is chosen arbitrarily. Then, he colors the edge adjacent to that vertex using the color opposite to the color of the spotted largest monochromatic star. The other edge is colored accordingly.

As an immediate consequence we obtain the following corollary.

Corollary 5 Let $k$ be an integer. In the balanced $k$-star avoidance game, we have $m_{S_{k}}=n^{\frac{2 k-2}{2 k-1}}$.

Next, we look at the balanced path game. The exact threshold remains outstanding, but can we give

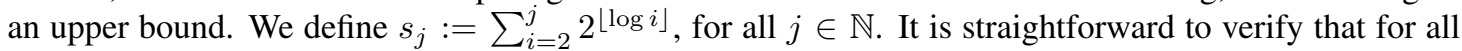
$m \in \mathbb{N}$, we have

(i) $4 s_{m}+4=s_{2 m+1}, m \geq 1$,

(ii) $2 s_{m}+2 s_{m-1}+4=s_{2 m}, m \geq 2$.

Using this property, we can give an upper bound for the balanced path game.

Proposition 6 For all $k \in \mathbb{N}, l \in \mathbb{N}$, the number of red $k$-paths after playing $\omega\left(n^{\frac{s_{l}}{s_{l}+1}}\right)$ moves of the balanced game is $\omega\left(n^{\left(s_{k}+1\right) \frac{s_{l}}{s_{l}+1}-s_{k}}\right)$ a.a.s.

In particular, this means that for all $l \in \mathbb{N}$, the number of red $l$-paths after playing $\omega\left(n^{\frac{s_{l}}{s_{l}+1}}\right)$ moves is $\omega(1)$ a.a.s., independent of Painter's strategy.

We can exhibit optimal strategies and give sharp bounds for 3-path and 4-path, $m_{P_{3}}=n^{4 / 5}$ and $m_{P_{4}}=n^{7 / 8}$. However, it seems to us that the method we use cannot be easily extended to the game of avoiding an arbitrary path. 


\section{References}

[1] József Beck. Deterministic graph games and a probabilistic intuition. Combin. Probab. Comput., 3(1):13-26, 1994.

[2] József Beck. Positional games and the second moment method. Combinatorica, 22(2):169-216, 2002. Special issue: Paul Erdős and his mathematics.

[3] József Beck. Ramsey games. Discrete Math., 249(1-3):3-30, 2002. Combinatorics, graph theory and computing (Louisville, KY, 1999).

[4] Ehud Friedgut, Yoshiharu Kohayakawa, Vojtěch Rödl, Andrzej Ruciński, and Prasad Tetali. Ramsey games against a one-armed bandit. Combin. Probab. Comput., 12(5-6):515-545, 2003. Special issue on Ramsey theory.

[5] Martin Marciniszyn, Reto Spöhel, and Angelika Steger. The online clique avoidance game on random graphs. Accepted for publication in the Proceedings of the 9th International Workshop on Randomization and Computation, 2005.

[6] Frank Plumpton Ramsey. On a problem of formal logic. Proceedings of the London Mathematical Society, 30:264-286, 1930.

[7] Vojtěch Rödl and Andrzej Ruciński. Threshold functions for Ramsey properties. J. Amer. Math. Soc., 8(4):917-942, 1995.

[8] Miloš Stojaković and Tibor Szabó. Positional games on random graphs. Random Structures Algorithms, 26(1-2):204-223, 2005.

[9] Van H. Vu. A large deviation result on the number of small subgraphs of a random graph. Combin. Probab. Comput., 10(1):79-94, 2001. 
